\title{
ON THE HEAT TRANSFER TO CONSTANT-PROPERTY LAMINAR BOUNDARY LAYER WITH POWER-FUNCTION FREE-STREAM VELOCITY AND WALL-TEMPERATURE DISTRIBUTIONS*
}

\author{
BY \\ ISAO IMAI** \\ Institute for Fluid Dynamics and Applied Mathematics, University of Maryland
}

Summary. The heat transfer to constant-property laminar boundary layer with porver-function variations of free stream velocity $\left(u_{1}=c x^{m}\right)$ and of temperature difference between wall and free stream $\left(T_{0}-T_{1}=b x^{n}\right)$ is studied by means of an improved version of the WKB method developed by the author. It is found that the local heattransfer coefficient $h$ can be approximately given in the form

$$
\frac{h x / k}{\left(u_{1} x / v\right)^{1 / 2}}=\frac{1}{(2-\beta)^{1 / 2}}\left[\frac{\Gamma(2 / 3)}{3^{2 / 3} \Gamma(4 / 3)}\left\{\frac{1}{2}+n(2-\beta)\right\}^{1 / 3}(\sigma \alpha)^{1 / 3}-\frac{\beta}{10 \alpha}\right],
$$

where $\beta=2 m /(m+1), \alpha$ is the non-dimensional velocity gradient at the wall (usually expressed as $\left.\alpha=f^{\prime \prime}(0)\right), \sigma$ is the Prandtl number, $k$ is the thermal conductivity, and $\nu$ is the kinematic viscosity.

As examples of application of the formula two cases of special interest, i.e. the Blasius flow and stagnation flow are considered for several values of $n$, and the results are compared with the purely numerical computations by Levy and by Chapman and Rubesin, as well as with the analytical approximate formulas obtained by Schuh, by Fettis and by Lighthill. It appears that the above formula is the most accurate among those hitherto proposed.

1. Introduction. The calculation of the heat transfer to the laminar boundary layer with arbitrary distributions of main flow velocity and wall temperature is very difficult even for the constant-property flow, because the problem is governed by a system of non-linear partial differential equations. But, as was shown by Fage and Falkner [1] the system of equations can be reduced to that of ordinary differential equations if both the main-stream velocity and the wall temperature are assumed to vary as power functions of distance from the start of the boundary layer, namely $u_{1}=c x^{m}$, $T_{0}-T_{1}=b x^{n}$. In fact, the momentum equation has been exactly solved by Hartree [2], and it remains only to solve the energy equation with variable coefficients given in terms of the Hartree solution. The exact treatment of the latter problem is naturally only possible by numerical means just as in the case of the momentum equation. Thus, Chapman and Rubesin [3] obtained the numerical solution for several values of $n$ for the case of the Blasius flow $(m=0)$ by use of the Runge-Kutta integration method, assuming the Prandtl number $\sigma$ to be 0.72. Later, Levy [4] carried out extensive numerical computations for a number of combinations of $m, n$, and $\sigma$ by reducing the differential equation to finite difference form and employing the IBM machine. From his numerical results, Levy proposed an empirical formula for the local heat-transfer coefficient.

*Received January 29, 1957. Most of the work was carried out while the author was at the Graduate School of Aeronautical Engineering, Cornell University. The author wishes to take this opportunity to thank Professor W. R. Sears, Director of the School, and his colleagues for their kind hospitality.

**Visiting Research Professor. On leave of absence from the University of Tokyo, Japan. 
In view of the considerable amount of labor involved in the numerical computations with three parameters $m, n$, and $\sigma$, Schuh [5] attempted to obtain the asymptotic solution of the energy equation when either $n$ or $\sigma$ is large so that the thermal boundary layer is very thin compared with the velocity boundary layer. Making further assumption that $\sigma / n \rightarrow 0$, he succeeded in obtaining the solution in a closed form using the Bessel function of order $1 / 3$ and hence a very compact formula for the local heat-transfer coefficient. Also he showed that the formula gives numerical results in good agreement with Levy's numerical computations. Dropping the second assumption $\sigma / n \rightarrow 0$, Punnis [6] and Fettis [7] succeeded in finding the solution in a closed form in terms of the confluent hypergeometric functions. Especially the latter author derived an explicit formula for the local heat-transfer coefficient, but he did not make any numerical discussion of the formula obtained.

In a paper which probably escaped the attention of the above-mentioned three authors, Lighthill [8] had developed a method of approximate calculation of heat transfer to the laminar boundary layer with arbitrary distributions of wall temperature and main-stream velocity by making the simplifying assumption that the velocity profile in the boundary layer can be approximated by its tangent at the wall. As an example, the method was applied to the Blasius flow and the result was found to be in good agreement with Chapman-Rubesin's numerical calculation.

On the same assumption as made by Lighthill, Tamaki [9] independently developed an approximate method of calculating thermal boundary layers, especially for the case of the Blasius flow; his formula for the locil heat-transfer coefficient is essentially the same as Lighthill's for this special case, but he treats a number of interesting cases using his formula and also extends the method so as to cover even the turbulent boundary layer. Further, he carried out numerical computations for our problem for the cases of $\beta=2 m /(m+1)=1,0.5,0,-0.14,-0.1988$ and the range of $n$ from -1.0 to 1.0 with $\sigma=0.7$.

In the present paper, the basic energy equation is solved by use of the improved version of the WKB method proposed by the author $[10,11,12]$ which has been shown by experience to give accurate results for various problems governed by the linear ordinary differential equation of the second order. The local heat-transfer coefficient is given in terms of algebraic functions of the three parameters $m, n$, and $\sigma$. Although the full expression obtained is somewhat complicated, it can be approximated by a rather simple expression. Applying the formulas, both full and simplified, to the cases of the Blasius flow $(m=0)$ and the stagnation flow $(m=1)$, it is found that they are in very good agreement with Levy's numerical computations.

2. Basic equations. For the laminar boundary layer on a body placed in an incompressible constant-property fluid, the continuity, momentum, and energy equations can be written as

$$
\begin{gathered}
\frac{\partial u}{\partial x}+\frac{\partial v}{\partial y}=0 \\
\rho\left(u \frac{\partial u}{\partial x}+v \frac{\partial u}{\partial y}\right)=-\frac{d p}{d x}+\mu \frac{\partial^{2} u}{\partial y^{2}}, \\
\rho C_{p}^{v}\left(u \frac{\partial T}{\partial x}+v \frac{\partial T}{\partial y}\right)=k \frac{\partial^{2} T^{\prime}}{\partial y^{2}}
\end{gathered}
$$


where $x$ and $y$ are measured parallel and perpendicular to the surface of the body, $u$ and $v$ are velocity components, $p$ is the pressure, $T$ is the temperature, $\rho$ is the density, $C_{p}$ is the specific heat at constant pressure, $k$ is the thermal conductivity, and $\mu$ is the viscosity. The boundary conditions are

$$
\begin{aligned}
& T=T_{0}(x), \quad u=v=0 \text { for } y=0 \\
& T=T_{1}, \quad u \rightarrow u_{1}(x) \quad \text { as } \quad y \rightarrow \infty
\end{aligned}
$$

where $T_{0}(x)$ is the surface temperature and $T_{1}$ and $u_{1}(x)$ are respectively the temperature and velocity of the main stream.

In the following, it will be assumed that

$$
\begin{aligned}
T_{0}-T_{1} & =b x^{n}, \\
u_{1} & =c x^{m},
\end{aligned}
$$

where $b, c, m$, and $n$ are constants. In this case it is known that the system of partial differential equations (2.1), (2.2), and (2.3) can be reduced to a pair of ordinary differential equations. Thus, introducing the variable

$$
\eta=\left(\frac{1+m}{2} \frac{u_{1}}{\nu x}\right)^{1 / 2} y
$$

and assuming that the stream function $\psi$ defined by

$$
u=\partial \psi / \partial y, \quad v=-\partial \psi / \partial x
$$

and the temperature $T$ can be expressed in the forms

$$
\begin{gathered}
\psi=\left(\frac{2}{1+m} \nu x u_{1}\right)^{1 / 2} f(\eta), \\
\frac{T-T_{1}}{T_{0}-T_{1}}=\theta(\eta),
\end{gathered}
$$

Eqs. (2.1), (2.2), and (2.3) are reduced to

$$
\begin{gathered}
f^{\prime \prime \prime}+f f^{\prime \prime}+\beta\left(1-f^{\prime 2}\right)=0, \\
\theta^{\prime \prime}+\sigma f \theta^{\prime}-\sigma n(2-\beta) f^{\prime} \theta=0,
\end{gathered}
$$

where $\beta=2 m /(m+1)$ and $\sigma=\mu C_{p} / k$ is the Prandtl number. The boundary conditions are

$$
f=f^{\prime}=0, \quad \theta=1 \text { for } \eta=0
$$

and

$$
f^{\prime} \rightarrow 1, \quad \theta \rightarrow 0 \quad \text { as } \quad \eta \rightarrow \infty .
$$

Equation (2.12) is well known as the Falkner-Skan equation and has been solved by Hartree [2] for several values of $\beta$. Hence Eq. (2.13) can be regarded as a linear differential equation of the second order with known variable coefficients.

3. The improved WKB method. For the integration of Eq. (2.13) it is convenient 
to make use of a refined version of the WKB method as developed by the present author $[10,11,12]$. Thus, consider a second-order linear differential equation

$$
\frac{d^{2} \Phi}{d x^{2}}+a^{2} P(x) \Phi=0, \quad a \rightarrow \infty,
$$

where $a$ is a parameter and $P(x)$ has the form

$$
P(x)=a_{1} x\left(1+b_{1} x+b_{2} x^{2}+\cdots\right), \quad a_{1} \neq 0 .
$$

Then, it can be shown that the solution of Eq. (3.1) can be expressed as

$$
\begin{aligned}
\Phi=P^{-1 / 4} \exp \left(Q / 4 a^{2}\right)\left\{C_{1}\right. & \exp \left[i\left(a z-Q_{1} / 2 a\right)\right] \\
+ & \left.C_{2} \exp \left[-i\left(a z-Q_{1} / 2 a\right)\right]\right\}+0\left(a^{-3}\right) \text { for } x \neq 0
\end{aligned}
$$

and

$$
\begin{aligned}
& \Phi=\left(\frac{\pi a}{2}\right)^{1 / 2} P^{-1 / 4} z^{1 / 8} \zeta\left\{C_{1} i^{i / 8} H_{1 / 3}^{(1)}(\omega)+C_{2} i^{-5 / /} H_{1 / 3}^{(2)}(\omega)\right\} \\
&+0\left(a^{-3}\right) \text { for } \quad x=0\left(a^{-2 / 3}\right),
\end{aligned}
$$

where

$$
\begin{gathered}
z=\int_{0}^{x} P^{1 / 2} d x, \\
Q=P^{-1 / 4} \frac{d^{2} P^{1 / 4}}{d z^{2}}=-P^{-3 / 4} \frac{d^{3} P^{-1 / 4}}{d x^{2}} \\
=-\left(\frac{5}{36} \frac{1}{z^{2}}+\lambda z^{-2 / 3}+\lambda_{0}+\lambda_{1} z^{2 / 3}+\cdots\right), \\
Q_{1}=\int_{0}^{2}\left(Q+\frac{5}{36} \frac{1}{z^{2}}\right) d z+\frac{5}{36} \frac{1}{z} \\
\omega=\kappa\left(\xi+\frac{1}{5} \lambda_{1} \kappa^{-2} \xi^{2}\right)^{3 / 2} \\
\zeta=\left(\xi-\frac{1}{5} \lambda_{1} \kappa^{-2} \xi^{2}\right)^{1 / 2} \\
\xi=z^{2 / 3}+\lambda^{-2}, \\
\kappa^{2}=a^{2}+\lambda_{0}
\end{gathered}
$$

and the constants $\lambda, \lambda_{0}, \lambda_{1}$ are given in terms of $a_{1}, b_{1}, \cdots$ as follows:

$$
\left.\begin{array}{rl}
\lambda= & -\frac{3}{35}\left(\frac{3}{2} a_{1}\right)^{-2 / 3}\left(5 b_{2}-3 b_{1}^{2}\right), \\
\lambda_{0}= & -\frac{4}{75} \frac{1}{a_{1}}\left(25 b_{3}-35 b_{1} b_{2}+14 b_{1}^{3}\right), \\
\lambda_{1}= & -\frac{27}{26950}\left(\frac{3}{2} a_{1}\right)^{-4 / 3}\left(6125 b_{4}-4350 b_{2}^{2}\right. \\
& \left.-9800 b_{1} b_{3}+12080 b_{1}^{2} b_{2}-3624 b_{1}^{4}\right) .
\end{array}\right\}
$$

In order to consider the behavior of $\Phi$ as $x \rightarrow 0$, it is convenient to use $J_{-1 / 3}(\omega)$ instead of $H_{1 / 3}^{(1,2)}(\omega)$. Thus, we have 


$$
\begin{aligned}
& H_{1 / 3}^{(1)}=\frac{2}{3^{1 / 2}} i^{2 / 3}\left(i^{-1 / 3} J_{1 / 3}-i^{1 / 3} J_{-1 / 3}\right), \\
& H_{1 / 3}^{(2)}=\frac{2}{3^{1 / 2}} i^{-2 / 3}\left(i^{1 / 3} J_{1 / 3}-i^{-1 / 3} J_{-1 / 3}\right)
\end{aligned}
$$

and

$$
\begin{array}{r}
P^{-1 / 4} z^{1 / 8} J_{1 / 3}(\omega) \\
=\left(\frac{3}{2} a_{1}\right)^{-1 / 8} \frac{2^{-1 / 3}}{\Gamma(4 / 3)} \lambda^{-5 / 3}\left[1-\left\{\frac{1}{5} b_{1}-\frac{2}{3} \lambda^{-1}\left(\frac{3}{2} a_{1}\right)^{1 / 3} \kappa^{2}\right\} x+\cdots\right], \\
P^{-1 / 4} z^{1 / 8} \zeta J_{-1 / 3}(\omega)=\left(\frac{3}{2} a_{1}\right)^{-1 / 8} \frac{2^{1 / 3}}{\Gamma(2 / 3)} \kappa^{-1 / 3}\left[1-\left\{\frac{1}{5} b_{1}\right.\right. \\
\left.\left.+\left(\frac{3}{4} \lambda^{2}+\frac{2}{15} \lambda_{1}\right)\left(\frac{3}{2} a_{1}\right)^{1 / 3} \kappa^{-2}\right\} x+\cdots\right] .
\end{array}
$$

4. Solution of the energy equation. In order to apply the above formulas, we have first to bring the basic equation (2.13) into the standard form (3.1). This can be done by putting

$$
\theta=\exp \left[-\frac{1}{2} \sigma \int_{0}^{\eta} f d \eta\right] \cdot \theta
$$

so that Eq. (2.13) becomes

$$
\theta^{\prime \prime}-P(\eta) \Theta=0,
$$

where

$$
P(\eta)=\frac{1}{4} \sigma^{2} f^{2}+\sigma\left\{\frac{1}{2}+n(2-\beta)\right\} f^{\prime} .
$$

Now, Eq. (4.3) should be expressed in a form similar to Eq. (3.2). For this purpose, differentiating Eq. (2.12) with respect to $\eta$ three times, putting $\eta=0$ each time, and using the boundary condition (2.14), we have

$$
\begin{aligned}
f=f^{\prime}=0, \quad f^{\prime \prime}=\alpha, \quad f^{\prime \prime \prime}=-\beta, \\
f^{\mathrm{IV}}=0, \quad f^{\mathrm{v}}=(2-\beta) \alpha^{2}, \quad f^{\mathrm{vI}}=2(2-3 \beta) \beta \alpha
\end{aligned}
$$

for $\eta=0$. Here we have put $f^{\prime \prime}(0)=\alpha$, whose values have been calculated by Hartree [2] for several values of $\beta$. Hence

$$
f=\frac{1}{2} \alpha \eta^{2}-\frac{1}{6} \beta \eta^{3}+\frac{1}{5 !}(2 \beta-1) \alpha^{2} \eta^{5}+\frac{1}{6 !} 2(2-3 \beta) \beta \alpha \eta^{6}+\cdots
$$

Substituting Eq. (4.5) into Eq. (4.3) we have

$$
\begin{aligned}
P(\eta)=A \eta\left[1-\frac{\beta}{2 \alpha} \eta+\left\{\frac{2 \beta-1}{24} \alpha+\right.\right. & \left.\frac{1}{16} \frac{\alpha^{2} \sigma^{2}}{A}\right\} \eta^{3} \\
& \left.+\left\{\frac{(2-3 \beta) \beta}{60}-\frac{1}{24} \frac{\alpha \beta \sigma^{2}}{A}\right\} \eta^{4}+\cdots\right],
\end{aligned}
$$


where

$$
A=\alpha \sigma\left\{\frac{1}{2}+n(2-\beta)\right\} .
$$

Comparison of Eqs. (3.1) and (3.2) with Eqs. (4.2) and (4.6) shows that

$$
\left.\begin{array}{c}
a=e^{i \times / 2}, \\
a_{1}=A, \quad b_{1}=-\frac{\beta}{2 \alpha}, \quad b_{2}=0, \\
b_{3}=\frac{\sigma^{2} \alpha^{2}}{16 A}-\frac{(1-2 \beta) \alpha}{24}, \quad b_{4}=\frac{(2-3 \beta) \beta}{60}-\frac{\sigma^{2} \alpha \beta}{24 A}
\end{array}\right\} .
$$

The formulas (3.3) and (3.4) have originally been obtained on the assumption that the parameter $a$ is very large. At first sight Eq. (4.8) might seem to conflict with this assumption. Since, however, the condition for the validity of the formulas is essentially that $|a z|$ should be large, the condition is readily seen to be satisfied if $A$ is large. This latter condition is satisfied if either the Prandtl number $\sigma$ or the exponent $n$ is large as was assumed by Schuh [5]. Furthermore, it should be mentioned that the formulas have been found to give accurate results in various cases of application even when $a$ is not very large.

Let us assume $A>0$. Then $P(\eta)>0$ for $\eta>0$ Consequently

$$
z=\int_{0}^{\eta} P^{1} \quad d_{\eta}>0
$$

so that

$$
i a z=-z<0 .
$$

Therefore, by the boundary condition (2.15), which can also be expressed as

$$
\theta \rightarrow 0 \text { as } z \rightarrow \infty
$$

we have to take $C_{2}=0$ in Eq. (3.3). Thus

$$
\Theta=C_{1} P^{-1 / 4} \exp \left[-\left(z+\frac{1}{2} Q_{1}+\frac{1}{4} Q\right)\right] \text { for } \eta \neq 0
$$

and

$$
\Theta=C_{1}\left(\frac{\pi i}{2}\right)^{1 / 2} P^{-1 / 4} z^{1 / 8} \zeta i^{5 / 8} H_{1 / 3}^{(1)}(\omega) \text { for } \quad \eta \doteqdot 0 .
$$

The constant $C_{1}$ can be determined by the boundary condition (2.14) as

$$
C_{1}=-(2 / \pi)^{1 / 2}[H(0)]^{-1} \text {. }
$$

Hence Eq. (4.12) becomes

$$
\Theta=H(\eta) / H(0) \text { for } \quad \eta \doteqdot 0,
$$

where

$$
H(\eta)=P^{-1 / 4} z^{1 / 6} \zeta i^{-2 / 3} H_{1 / 3}^{(1)}(\omega) .
$$

Now the heat flow rate at the wall is given by

$$
q_{0}=-k\left(\frac{\partial T}{\partial y}\right)_{\nu=0}=-k \frac{T_{0}-T_{1}}{(2-\beta)^{1 / 2}}\left(\frac{u_{1}}{\nu x}\right)^{1 / 2}\left(\frac{d \theta}{d \eta}\right)_{\eta=0} .
$$


Therefore, if the local heat-transfer coefficient $h$ is defined by

$$
h=\frac{q_{n}}{T_{0}-T_{1}},
$$

we have

$$
\frac{h x / k}{\left(u_{1} x / \nu\right)^{1 / 2}}=-(2-\beta)^{-1 / 2}\left(\frac{d \theta}{d \eta}\right)_{\eta=0} .
$$

But, by Eqs. (4.1) and (4.14)

$$
\left(\frac{d \theta}{d \eta}\right)_{\eta=0}=\left(\frac{d \theta}{d \eta}\right)_{\eta=0}=\frac{H^{\prime}(0)}{H(0)} .
$$

Now, combining Eqs. (3.6), (3.8), and (3.9), we have

$$
\begin{gathered}
H(0)=-{\frac{2}{3^{2 / 3}}}^{11 / 6} \frac{\left[\left(1-\lambda_{0}\right) A\right]^{-1 / 6}}{\Gamma(2 / 3)}\left\{1+\frac{2^{-2 / 3} \Gamma(2 / 3)}{\Gamma(4 / 3)} \lambda\left(1-\lambda_{0}\right)^{-2 / 3}\right\}, \\
\frac{H^{\prime}(0)}{H(0)}=-\frac{1}{5} b_{1}-\left(\frac{2}{3}\right)^{2 / 3}\left[\left(1-\lambda_{0}\right) A\right]^{1 / 3} \frac{1-c\left[(9 / 8) \lambda^{2}+\frac{1}{5} \lambda_{1}\right]\left(1-\lambda_{0}\right)^{-4 / 3}}{c+\lambda\left(1-\lambda_{0}\right)^{-2 / 3}},
\end{gathered}
$$

where

$$
c=\frac{2}{\Gamma^{\prime}(2 / 3)}=1.04682
$$

and the constants $b_{1}, \lambda, \lambda_{0}, \lambda_{1}$ are given by Eqs. (3.12) and (4.9) as follows

$$
\left.\begin{array}{l}
\lambda=\frac{9}{35}\left(\frac{3}{2} A\right)^{-2 / 3} b_{1}^{2}, \\
\lambda_{0}=-\frac{4}{75} A^{-1}\left(25 b_{3}+14 b_{1}^{3}\right), \\
\lambda_{1}=-\frac{27}{26950}\left(\frac{3}{2} A\right)^{-4 / 3}\left(6125 b_{1}-9800 b_{1} b_{3}-3624 b_{1}^{4}\right),
\end{array}\right\}
$$

where

$$
\left.\begin{array}{l}
b_{1}=-\frac{\beta}{2 \alpha}, \quad b_{3}=-\frac{(1-2 \beta) \alpha}{2+}+\frac{\sigma^{2} \alpha^{2}}{16 A}, \\
b_{4}=\frac{(2-3 \beta) \beta}{60}-\frac{\sigma^{2} \alpha \beta}{24 A}, \quad A=\left\{\frac{1}{2}+n(2-\beta)\right\} \sigma \alpha .
\end{array}\right\}
$$

If it is assumed that $A$ is very large while $b_{1}, b_{3}$, and $b_{4}$ are $0(1)$ (this is true if $n$ is very large so that $A=0(n)$ ), Eqs. (4.23) give

$$
\lambda=0\left(A^{-2 / 3}\right), \quad \lambda_{0}=0\left(A^{-1}\right), \quad \lambda_{1}=0\left(A^{-4 / 3}\right) .
$$

Hence Eq. (4.21) can be approximated by

$$
\frac{H^{\prime}(0)}{H(0)}=\frac{\beta}{10 \alpha}-\left(\frac{1}{3}\right)^{2 / 3} \frac{\Gamma(2 / 3)}{\Gamma(4 / 3)} A^{1 / 3}+0\left(A^{-1 / 3}\right) .
$$


5. Comparison with previous investigations. The temperature distribution in the boundary layer is given by Eqs. (4.1), (4.11), (4.13), and (4.14) in the form

$$
\theta(\eta)=-\left(\frac{2}{\pi}\right)^{1 / 2} \frac{P^{-1 / 4}}{H(0)} \exp \left[-\left(\frac{1}{2} \sigma \int_{0}^{\eta} f d \eta+z+\frac{1}{2} Q_{1}+\frac{1}{4} Q\right)\right] \text { for } \eta \neq 0
$$

and

$$
\theta(\eta)=\frac{H(\eta)}{H(0)} \exp \left[-\frac{1}{2} \sigma \int_{0}^{\eta} f d \eta\right] \text { for } \eta \doteqdot 0 .
$$

In the following we shall restrict ourselves to the heat-transfer coefficient $h$, which is given by

$$
\frac{h x / \dot{k}}{\left(u_{1} x / \nu\right)^{1 / 2}}=-(2-\beta)^{-1 / 2} \frac{H^{\prime}(0)}{H(0)} \text {. }
$$

Here $H^{\prime}(0) / H(0)$ is given by Eq. (4.21) or approximately by Eq. (4.25).

First let us compare our result with the results of previous investigators.

Schuh [5] considers that the thickness of the thermal boundary layer becomes small compared to the velocity boundary layer if either $n$ or $\sigma$ becomes large, and that it would then be sufficient to replace the velocity profile by its tangent at the wall, since, for calculating the temperature field, only that part of the velocity profile is important which lies within the thermal boundary layer. Thus he puts

$$
d f / d \eta=\alpha \eta
$$

so that Eq. (2.13) reduces to

$$
\theta^{\prime \prime}+\frac{1}{2} \alpha \sigma \eta^{2} \theta^{\prime}-\sigma n \alpha(2-\beta) \eta \theta=0
$$

which, on putting

$$
\zeta=n^{1 / 3} \eta
$$

becomes

$$
\frac{d^{2} \theta}{d \zeta^{2}}+\frac{\sigma}{n} \frac{\alpha}{2} \zeta^{2} \frac{d \theta}{d \zeta}-\sigma \alpha(2-\beta) \zeta \theta=0 .
$$

Further he assumes that

$$
\sigma / n \rightarrow 0
$$

so that Eq. (5.7) simplifies itself to

$$
d^{2} \theta / d \zeta^{2}-\sigma \alpha(2-\beta) \zeta \theta=0 .
$$

This can be integrated in terms of the Bessel function of order $1 / 3$. The final result for the heat-transfer coefficient is

$$
\frac{h x / k}{\left(u_{1} x / \nu\right)^{1 / 2}}=\frac{\Gamma(2 / 3)}{3^{2 / 3} \Gamma(4 / 3)} \frac{(\sigma \alpha n)^{1 / 3}}{(2-\beta)^{1 / 6}} .
$$

Next he tried to obtain an asymptotic solution of Eq. (5.7) which is valid for $\sigma \rightarrow \infty$ with no restriction on $n$. For this purpose, he introduced a new variable $\xi$ defined by

$$
\xi=(\sigma \alpha)^{1 / 3} \eta
$$


to write Eq. (5.7) in the form

$$
d^{2} \theta / d \xi^{2}+\frac{1}{2} \xi^{2} d \theta / d \xi-\epsilon \xi \theta=0
$$

with

$$
\epsilon=n(2-\beta) \text {. }
$$

However, he could not find any solution of Eq. (5.12) in a closed form and made no attempt to solve it numerically. As examples of application of the formula (5.10), he calculated the values of $(h x / k)\left(u_{1} x / \nu\right)^{-1 / 2}$ for the Blasius flow $(\beta=0)$ and for the stagnation flow $(\beta=1)$ for several values of $n$ and compared the results with the purely numerical calculations by Chapman and Rubesin [3] and by Levy [4].

Later, Punnis [6] and Fettis [7] independently showed that Eq. (5.12) can be integrated in a closed form using confluent hypergeometric functions. Furthermore, the latter gave an explicit expression for the heat-transfer coefficient at the wall. His result for the temperature distribution function $\theta$ is expressed as

$$
\theta=\frac{\Gamma(1+2 \epsilon / 3)}{\Gamma(1 / 3)} \xi^{-1} \exp \left(-\xi^{3 / 12}\right) W_{-(1+2 \epsilon / 3), 1 / 8}\left(\xi^{3} / 6\right)
$$

where $W_{k, m}(t)$ denotes the Whittaker function and $\xi$ is defined by Eq. (5.11). The heat-transfer coefficient is given by the formula

$$
\frac{h x / k}{\left(u_{1} x / \nu\right)^{1 / 2}}=-\frac{(\sigma \alpha / 6)^{1 / 3}}{(2-\beta)^{1 / 2}} \frac{\Gamma(1+2 \epsilon / 3) \Gamma(-1 / 3)}{\Gamma(2 / 3+2 \epsilon / 3) \Gamma(1 / 3)} .
$$

Now it should be mentioned that Lighthill [8] had previously obtained a general formula which gives the heat transfer at the wall in terms of the skin friction for arbitrary main-stream velocity and wall-temperature distributions, based on the simplifying assumption adopted by Fage and Falkner [1]:

$$
u=(1 / \mu) \tau(x) y
$$

where $\tau(x)$ is the skin friction so that $\tau(x)=\mu(\partial u / \partial y)_{\nu=0}$. This assumption is of course the same as Eq. (5.4). Lighthill's formula can be written in our notation as follows.

$$
q_{0}(x)=k\left(\frac{\sigma \rho}{9 \mu^{2}}\right)^{1 / 3} \frac{[\tau(x)]^{1 / 2}}{\Gamma(4 / 3)} \int_{0}^{x}\left(\int_{x_{1}}^{x}[\tau(z)]^{1 / 2} d z\right)^{-1 / 3} d T_{0}\left(x_{1}\right)
$$

Lighthill himself applied this formula to the case of the Blasius flow in order to test its accuracy.

Now the formula (5.17) will be applied to our problem defined by Eqs. (2.6) and (2.7). First we have, by Eqs. (2.8), (2.9), and (2.10),

$$
\tau(x)=\mu\left(\frac{\partial u}{\partial y}\right)_{y=0}=\mu u_{1} f^{\prime \prime}(0)\left(\frac{1+m}{2} \frac{u_{1}}{\nu x}\right)^{1 / 2} .
$$

Introducing Eqs. (5.18), (2.6), and (2.7) into Eq. (5.17) and making some calculation, we obtain

$$
\frac{q_{0}(x)}{\left(T_{0}-T_{1}\right) k\left(u_{1} / \nu x\right)^{1 / 2}}=-\frac{(\sigma \alpha / 6)^{1 / 3}}{(2-\beta)^{1 / 2}} \frac{\Gamma(1+2 \epsilon / 3)}{\Gamma(2 / 3+2 \epsilon / 3)} \frac{\Gamma(-1 / 3)}{\Gamma(1 / 3)}
$$


which is nothing other than the formula (5.15). Therefore, it may be stated that Fettis' result is included in Lighthill's general formula. This is perhaps what should have been expected, because both results have been obtained under the same simplifying assumption about the velocity profile. Further it may be remarked that some idea of the accuracy of Lighthill's formula (5.17) may be obtained by comparison of the result (5.19) with our and other calculations.

Extensive numerical computations have been performed by Levy [4] by reducing Eq. (2.13) to finite difference form and employing an electronic IBM machine; the results cover a range of $n$ from -2.5 to 4.0 and $\sigma$ from 0.70 to 20.0 for four values of $m(4,1,0$, -0.0904). For the range of the calculations, it was found that the local heat-transfer coefficient can, with the exception of large negative $n$ values, be expressed within 5 per cent as

$$
\frac{h x / k}{\left(u_{1} x / \nu\right)^{1 / 2}}=B(m, n) \sigma^{\gamma},
$$

where the function $B(m, n)$ can be approximated by

$$
B(m, n)=0.57\left(\frac{2 m}{m+1}+0.205\right)^{0.104}\left(\frac{2 n}{m+1}+1\right)^{0.37+0 \cdot 12 m /(m+1)}
$$

and the exponent $\gamma$ of the Prandtl number varies as shown in the following table:
$\beta$
1.6
1.0
0
$\gamma$
0.367
0.355
0.327
0.254

6. Blasius flow. For the case of the Blasius flow we have $\beta=0$, so that Eqs. (4.24) and (4.23) give

$$
\begin{aligned}
& b_{1}=0, \quad b_{3}=\frac{\sigma^{2} \alpha^{2}}{16 A}-\frac{\alpha}{24}, \quad b_{4}=0 \\
& \lambda=0, \quad \lambda_{0}=-\frac{1}{12} \frac{\sigma^{2} \alpha^{2}}{A^{2}}+\frac{1}{18} \frac{\alpha}{A}, \quad \lambda_{1}=0 .
\end{aligned}
$$

Hence Eqs. (5.3) and (4.21) become

$$
\frac{h x / k}{\left(u_{1} x / \nu\right)^{1 / 2}}=\frac{1}{2^{1 / 2}} \frac{\Gamma(2 / 3)}{3^{2 / 3} \Gamma^{\prime}(4 / 3)} A^{1 / 3}\left(1-\frac{1}{18} \frac{\alpha}{A}+\frac{1}{12} \frac{\sigma^{2} \alpha^{2}}{A^{2}}\right)^{1 / 3}
$$

which can be approximated by

$$
\frac{h x / k}{\left(u_{1} x / \nu\right)^{1 / 2}}=\frac{1}{2^{1 / 2}} \frac{\Gamma(2 / 3)}{3^{3 / 3} \Gamma(4 / 3)} A^{1 / 3},
$$

where

$$
A=\left(\frac{1}{2}+2 n\right) \sigma \alpha, \quad \alpha=0.4696 .
$$

In this case, Schuh's formula (5.10) becomes

$$
\frac{h x / k}{\left(u_{1} x / \nu\right)^{1 / 2}}=\frac{\Gamma(2 / 3)(n \sigma \alpha)^{1 / 3}}{2^{1 / 6} 3^{2 / 3} \Gamma(4 / 3)}
$$


and Fettis' formula (5.15) becomes

$$
\frac{h x / k}{\left(u_{1} x / \nu\right)^{1 / 2}}=2^{-1 / 2} 6^{-1 / 3} \frac{\Gamma(2 / 3) \Gamma(1+4 n / 3)}{\Gamma(4 / 3) \Gamma(2 / 3+4 n / 3)}(\sigma \alpha)^{1 / 3}
$$

which is essentially the same as the formula given by Lighthill. Table 1 shows the values of $(h x / k)\left(u_{1} x / \nu\right)^{-1 / 2}$ for several values of $n$ as calculated by the various formulas. In Table 1 the first column shows the values calculated by Chapman and Rubesin by the use of the Runge-Kutta integration method. The Levy values were computed by the present author from Levy's values for $\sigma=0.7$ by using Levy's empirical formula (5.20).

TABLE 1

Local heat-transfer coefficients for Blasius flow $(\beta=0)$. Prandtl number $=0.72$.

\begin{tabular}{|c|c|c|c|c|c|c|}
\hline$n$ & $\begin{array}{l}\text { Chapman- } \\
\text { Rubesin }\end{array}$ & Levy & $\begin{array}{l}\text { Lighthill } \\
\text { Fettis }\end{array}$ & Schuh & Imai & Imai* \\
\hline 0 & 0.296 & 0.2901 & 0.304 & 0 & 0.3011 & 0.2850 \\
\hline 1 & 0.489 & 0.4814 & 0.490 & 0.4524 & 0.4845 & 0.4874 \\
\hline 2 & 0.597 & 0.5865 & 0.594 & 0.5700 & 0.5903 & 0.5929 \\
\hline 3 & 0.684 & 0.6634 & 0.671 & 0.6525 & 0.6680 & 0.6702 \\
\hline 4 & 0.744 & 0.7258 & 0.734 & 0.7182 & 0.7310 & 0.7329 \\
\hline 5 & 0.799 & - & 0.787 & 0.7737 & 0.7847 & 0.7864 \\
\hline 10 & 1.006 & - & 0.987 & 0.9747 & 0.9817 & 0.9829 \\
\hline
\end{tabular}

The Lighthill values have been reproduced from his paper [8] and are the same as those given by Eq. (6.5). The "Imai" values are those calculated by use of the author's full expression (6.1), while the "Imai"" values are those calculated by the simplified expression (6.2). It will be seen that the values calculated by the author's formulas, both full and simplified, are in agreement with those computed by Levy within 1 per cent, except for the case of $n=0$, where the difference is less than 2 per cent and 4 per cent, respectively, for the full and the simplified formula. By performing detailed examination of the accuracy of his numerical computations, Levy has estimated the accuracy of his results to be within 2 per cent. Therefore, our formula (6.1) and (6.2) may be considered to be perfectly satisfactory. Moreover, because of the simplicity, the simplified formula may be especially recommended for practical purposes.

Further it may be observed that Lighthill and Fettis' formula is better than Schuh's, which, however, is fairly good except for the case of $n=0$. This rather high accuracy of the formulas of Schuh and of Lighthill and Fettis is naturally due to the fact that the simplifying assumption $u \propto y$ is very accurate for the Blasius flow.

7. Stagnation flow. Similar comparison is made for the case of the stagnation flow $(\beta=1)$, taking $\sigma=1$ and 10 .

Table 2 shows the case of $\sigma=1$. Here the "Levy" values have been reproduced from his paper, while the "Levy*" values are those computed by the present author using the empirical formula (5.21). The "Fettis" and "Schuh" values were also computed by the author by use of Eqs. (5.10) and (5.15) respectively. The "Imai" and "Imai*" values are those calculated by the author's formulas (4.19) and (4.23) respectively.

It will be seen that both the Fettis and Schuh values are not in very good agreement with the Levy values. It is interesting to note that the Schuh values are better than the Fettis values except for small values of $n$, in spite of the fact that the former is an approxi- 
TABLE 2

Local heat-transfer coefficients for stagnation flow $(\beta=1)$. Prandtl number $=1$.

$\begin{array}{lcccccc}n & \text { Levy } & \text { Levy* } & \text { Fettis } & \text { Schuh } & \text { Imai } & \text { Imai* } \\ 0 & 0.5603 & 0.581 & 0.661 & 0 & 0.584 & 0.539 \\ 0.25 & 0.6345 & 0.640 & 0.735 & 0.492 & 0.645 & 0.629 \\ 0.5 & 0.6979 & 0.692 & 0.799 & 0.620 & 0.705 & 0.701 \\ 1 & 0.8116 & 0.783 & 0.905 & 0.782 & 0.809 & 0.814 \\ 2 & 0.9647 & 0.932 & 1.065 & 0.985 & 0.971 & 0.980 \\ 3 & 1.089 & 1.055 & 1.189 & 1.127 & 1.096 & 1.106 \\ 4 & 1.192 & 1.161 & 1.292 & 1.241 & 1.200 & 1.209 \\ 5 & - & 1.256 & 1.382 & 1.337 & 1.289 & 1.299 \\ 10 & - & 1.630 & 1.693 & 1.684 & 1.622 & 1.630\end{array}$

mation to the latter. As has been remarked by Schuh, this is probably due to the fact that the following two influences cancel each other: (a) approximating the velocity profile by its tangent at the wall increases $h$ for $\beta>0$; (b) neglecting the middle term in Eq. (5.7) reduces $h$.

On the other hand, both the simplified and full formulas of the author give results in satisfactory agreement with the Levy values. It will be observed that the approximate "Imai*" values are even better than the "Levy*" values calculated by the rather complicated empirical formula.

Titule 3 shows a similar comparison for the cace of rather high Prandtl number of $\sigma=10$. The situation is very similar to the above case of $\sigma=1$. Here the superiority of the Schuh formula to the Fettis for $n>1$ is rather more conspicuous. However, our simplified formula is seen to be in closest agreement with Levy's computation.

8. Conclusion. Summarizing the result of the above comparison it may be stated that our approximate formula

$$
\frac{h x / k}{\left(u_{1} x / \nu\right)^{i / 2}}=\frac{1}{(2-\beta)^{1 / 2}}\left[\left(\frac{1}{3}\right)^{2 / 3} \frac{\Gamma(2 / 3)}{\Gamma(4 / 3)}\left\{\frac{1}{2}+n(2-\beta)\right\}^{1 / 3}(\sigma \alpha)^{1 / 3}-\frac{\beta}{10 \alpha}\right]
$$

TABLE 3

Local heat-transfer coefficients for stagnation flow $(\beta=1)$. Prandtl number $=10$.

$\begin{array}{lccccc}n & \text { Levy } & \text { Levy* } & \text { Fettis } & \text { Schuh } & \text { Imai* } \\ 0 & 1.286 & 1.316 & 1.424 & 0 & 1.255 \\ 0.25 & 1.451 & 1.449 & 1.584 & 1.061 & 1.449 \\ 0.5 & 1.590 & 1.567 & 1.721 & 1.337 & 1.603 \\ 1 & 1.818 & 1.773 & 1.949 & 1.684 & 1.847 \\ 2 & 2.159 & 2.111 & 2.295 & 2.122 & 2.204 \\ 3 & 2.418 & 2.389 & 2.562 & 2.429 & 2.476 \\ 4 & 2.630 & 2.629 & 2.784 & 2.673 & 2.699 \\ 5 & - & 2.844 & 2.976 & 2.880 & 2.891 \\ 10 & - & 3.691 & 3.649 & 3.628 & 3.606\end{array}$

is the best of the formulas hitherto proposed in view of the fact that it shows the effect of the parameters $\beta$ (or $m$ ), $n$ and $\sigma$ in a simple functional form. Especially it will be interesting to note that the exponent of $\sigma$ is invariably $1 / 3$ and that the effect of the curvature of the velocity profile at the wall appears as an additive constant $-\beta / 10 \alpha$ in the formula. 


\section{REFERENCES}

1. A. Fage and V. M. Falkner, Relation between heat transfer and surface friction for laminar flow, A.R.C.R. \& M., No. 1408, London, 1931

2. D. R. Hartree, On an equation occurring in Falkner and Skan's approximate treatment of the equation of the boundary layer, Proc. Cambridge Phil. Soc. 33, 223-239 (1937)

3. D. R. Chapman and M. W. Rubesin, Temperature and velocity profiles in the compressible laminar boundary layer with arbitrary distribution of surface temperature, J. Aeronaut. Sci. 16, No. 9, 547-565 (Sept. 1949)

4. S. Levy, Heat transfer to constant-property laminar boundary-layer flows with power-function freestream velocity and wall-temperature variation, J. Aeronaut. Sci. 19, No. 5, 341-348 (May 1952)

5. H. Schuh, On asymptotic solutions for the heat transfer at varying wall temperatures in a laminar boundary layer with Hartree's velocity profiles, J. Aeronaut. Sci. 20, No. 2, 146-147 (Feb. 1953)

6. B. Punnis, $A$ remark on "On asymptotic solutions for the heat transfer at varying wall temperatures in a laminar boundary layer with Hartree's velocity profiles," J. Aeronaut. Sci. 20, No. 7, 505 (July 1953)

7. H. E. Fettis, On a differential equation occurring in the theory of heat flow in boundary layers with Hartree's velocity profiles, J. Aeronaut. Sci. 21, No. 2, 132-133 (Feb. 1954)

8. M. J. Lighthill, Contributions to the theory of heat transfer through a laminar boundary layer, Proc. Roy. Soc. London A202, No. 1070, 359-377 (1950)

9. H. Tamaki, Studies of surface heat transfer based on the boundary layer theory, (in Japanese), Rept. Inst. Ind. Sci., Univ. of Tokyo 1, No. 8, 239-273 (March 1951)

10. I. Imai, On a refinement of the WKB method, Phys. Rev. 74, 113 (July 1, 1948)

11. I. Imai, Asymptotic solutions of ordinary linear differential equations of the second order, Phys. Rev. 80, 1112 (Dec. 15, 1950)

12. I. Imai, $A$ refinement of the WKB method and its application to the electromagnetic $w \ldots$. Trans. AP-4, No. 3, 233-239 (July 1956); proceedings of the Symposium on Electromagnetir Wave Theory held June 20-25, 1955, at the University of Michigan 